\section{DISSECTING THE SPATIAL HETEROGENEITY OF SARS- COV-2-INFECTED TUMOUR MICROENVIRONMENT REVEALS A LYMPHOCYTE-DOMINANT IMMUNE RESPONSE IN A HBV-ASSOCIATED HCC PATIENT WITH COVID-19 HISTORY}

${ }^{1}$ Benedict Tan*, ${ }^{2}$ Yi Yang, ${ }^{2}$ Chun Chau Lawrence Cheung, 'Denise Goh, ${ }^{1}$ Mai Chan Lau, ${ }^{1}$ Xinru Lim, ${ }^{1}$ Jeffrey Lim, ${ }^{1}$ Li Wen Justina Nadia Lee, ${ }^{1}$ Tracy Tien, ${ }^{2}$ Shirin Kalimuddin, ${ }^{3}$ Wai Meng David Tai, ${ }^{2}$ Jenny Low, ${ }^{3}$ Cedric Chuan Young Ng, ${ }^{4}$ Wei Qiang Leow, ${ }^{4}$ Thuan Tong Tan, ${ }^{4}$ Tony Lim, ${ }^{5}$ Jin Liu, ${ }^{1}$ Joe Yeong. ${ }^{1} I M C B, A * S T A R$, Singapore, Singapore; ${ }^{2}$ DukeNUS Medical School, Singapore, Singapore; ${ }^{3}$ National Cancer Centre Singapore, Singapore, Singapore; ${ }^{4}$ Singapore General Hospital, Singapore, Singapore; ${ }^{5}$ Duke-NUS, Singapore, Singapore

Background We previously reported the presence of SARSCoV-2 RNA in the hepatic tissues of recovered patients ${ }^{1}$ but the spatial immune profile of SARS-CoV-2 infection remains poorly understood. To address this, here we performed deep spatial profiling in tumour-adjacent normal hepatic tissue from a HBV-associated hepatocellular carcinoma (HCC) patient with history of COVID-19.

Methods We obtained tissue from curative resection of a HCC patient 85 days post-recovery from COVID-19. Spatial immune profiling was performed by multiplex immunohistochemistry (mIHC) ${ }^{2}$ and more deeply using the Visium spatial transcriptomics platform complemented with signatures derived from single-cell RNA sequencing (scRNA-seq) and published signatures.

Results SARS-CoV-2 nucleocapsid and spike proteins were detected in a tumour-adjacent normal hepatic section in a spatially-restricted pattern (figure $1 \mathrm{~A}$ and $\mathrm{B}$ ) and higher abundance of lymphocytes but not macrophages were observed in regions with virus detection (figure 1C). We employed spatial transcriptomics and scRNA-seq to further characterize the immune microenvironment of SARS-CoV-2 post-infection. Unsupervised clustering and automatic annotation ${ }^{3}$ of Visium spots revealed that the distribution of SARS-CoV-2 viral proteins partially coincided with a memory T-cell signature (figure 1D). Quantification of Visium transcriptomic spots using an independent transcriptomic signature based on genes differentially upregulated in immune cells in SARS-CoV-2 infection ${ }^{4}$ (figure 1E) resulted in an enrichment pattern similar to the SARS-CoV-2 protein distribution. Additionally, a signature derived from scRNA-seq of hepatic tumour-infiltrating lymphocytes after ex vivo peptide stimulation using a pool of SARSCoV-2 peptides showed a strongly associated distribution, in line with a SARS-CoV2-specific immune response ${ }^{5}$ whereas that from using a pool of HBV peptides resulted in an anticorrelated distribution (figure $1 \mathrm{~F}$ ). These illustrate the ability of spatial transcriptomics to quantify with microenvironmentlevel resolution the SARS-CoV-2-specific immune response. Recapitulating the mIHC protein data, deconvolution of immune populations ${ }^{6}$ revealed marked spatial associations between SARS-CoV-2 viral presence and the distributions of lymphocytes but not of macrophages (figure 1G).

Conclusions We believe this is the first deep profiling report of non-post-mortem samples which adopts a multi-modal approach combining mIHC, spatial transcriptomics, and transcriptomic signatures derived from scRNA-seq to interrogate the in situ immune response to viral infection. Applying this to SARS-CoV-2 infection, we detected tissue spatial heterogeneity in viral presence and an associated lymphocyte-dominant immune response in the COVID-19-recovered patient, in contrast to post-mortem observations of scarce lymphocytes in cases of severe COVID-19. ${ }^{7}$ Ongoing work including further validation of the findings in local and overseas cohorts and their correlation with patient clinical outcomes.
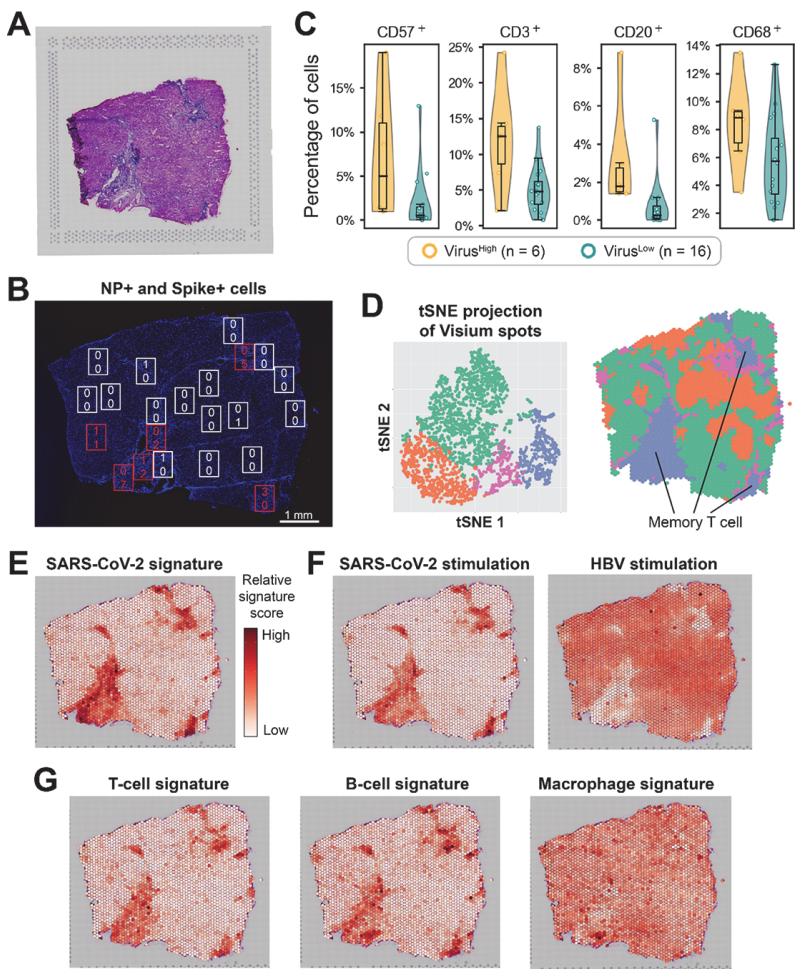

Abstract 626 Figure 1 Spatial heterogeneity of SARS-CoV-2 infection uncovers an association with a dominant lymphocytic response

\section{REFERENCES}

1. Cheung $C C L$, et al. Residual SARS-CoV-2 viral antigens detected in GI and hepatic tissues from five recovered patients with COVID-19. Gut, p. gutjnl-2021324280, 2021. doi: 10.1136/gutjnl-2021-324280.

2. Lim JCT, et al. An automated staining protocol for seven-colour immunofluorescence of human tissue sections for diagnostic and prognostic use. Pathology (Phila.) 2018;50(3):333-341. doi: 10.1016/j.pathol.2017.11.087.

3. Shao X, Liao J, Lu X, Xue R, Ai N, Fan X. scCATCH: automatic annotation on cell types of Clusters from Single-Cell RNA Sequencing Data. iscience 2020;23 (3):100882, doi: 10.1016/j.isci.2020.100882.

4. Lee JS, et al. Immunophenotyping of COVID-19 and influenza highlights the role of type I interferons in development of severe COVID-19. Sci Immunol 2020;5 (49):p.eabd1554. doi: 10.1126/sciimmunol.abd1554.

5. Schub D, et al. High levels of SARS-CoV-2-specific T cells with restricted functionality in severe courses of COVID-19. JCI Insight 2020;5(20):p.e142167. doi: 10.1172/jci.insight.142167.

6. Newman $A M$, et al. Robust enumeration of cell subsets from tissue expression profiles. Nat Methods 2015;12(5):453-457. doi: 10.1038/nmeth.3337.

7. Wang $Y$, et al. SARS-CoV-2 infection of the liver directly contributes to hepatic impairment in patients with COVID-19. J Hepatol 2020;73(4):807-816. doi: 10.1016/j.jhep.2020.05.002.

Ethics Approval This study was approved by the SingHealth Centralised Institutional Review Board (reference number: 2019/2653)

http://dx.doi.org/10.1136/jitc-2021-SITC2021.626 